\title{
Periodic dynamic systems for infected hosts and mosquitoes
}

\section{Sistemas dinâmicos periódicos para hospedeiros e mosquitos infectados}

\author{
W. M. Oliva and E. M. Sallum \\ Departamento de Matemática Aplicada do Instituto Superior Técnico da Universidade Técnica de \\ Lisboa. Portugal (W.M.O.), Instituto de Matemática e Estatística da Universidade de São Paulo. São \\ Paulo, SP - Brasil (E.M.S.)
}

\begin{abstract}
A mathematical model for the purpose of analysing the dynamic of the populations of infected hosts anf infected mosquitoes when the populations of mosquitoes are periodic in time is here presented. By the computation of a parameter $\lambda$ (the spectral radius of a certain monodromy matrix) one can state that either the infection peters out naturally) $(\lambda \leq 1)$ or if $\lambda>1$ the infection becomes endemic. The model generalizes previous models for malaria by considering the case of periodic coefficients; it is also a variation of that for gonorrhea. The main motivation for the consideration of this present model was the recent studies on mosquitoes at an experimental rice irrigation system, in the South-Eastern region of Brazil.
\end{abstract}

Malaria, epidemiology. Culicidae. Population dinamics. Ecology, vectors.

\section{Resumo}

Desenvolveu-se um modelo matemático para analisar a dinâmica das populações de indivíduos e mosquitos infectados quando as populações de mosquitos são periódicas no tempo. Pela determinação de um parâmetro $\lambda$ (o raio espectral de uma matriz de monodromia) pode-se estabelecer que a infecção termina naturalmente $(\lambda \leq 1)$ ou se $\lambda>1$ que a infecção torna-se endêmica. $O$ modelo generaliza, para o caso de coeficientes periódicos, modelos anteriores para malária; como também é uma variação de modelo para a gonorréia. A principal motivação para a consideração do modelo proposto foram os recentes estudos sobre mosquitos numa estação experimental de arroz irrigado, na região Sudeste do Brasil.

Malária, epidemiologia. Culicidae. Dinâmica populacional. Ecologia de vetores. 


\section{INTRODUCTION}

A model was constructed for the purpose of analysing the dynamic of the populations of infected hosts and infected mosquitoes, when the populations of mosquitoes are periodic in time. A deterministic estimator $\lambda$ was established to predict whether the infection will peter out naturally (when $\lambda \leq 1$ ) or, if $\lambda>1$, the infection will become endemic (see Appendix). In the model, incubation and immunity are neglected.

The special case of one patch of hosts and two patches of mosquitoes are considered and the main results concerning the dynamics are given (see Theorems A and B). The estimator $\lambda$ is the spectral radius of the monodromy matrix $C$, corresponding to the periodic matrix $A(t)$ of the linear system associated with the complete system (1). It is a non trivial matter to compute the matrix $C$ as also to discover its spectral radius $\lambda$.

The case of one patch of hosts and one (resp. two) patch(es) of mosquitoes is studied and it is assumed that the populations of mosquitoes behave according to periodic step functions. The monodromy matrix $C$ is the product of exponentials of computable matrices; moreover, using suitable changes of coordinates, it was possible to obtain explicitly all the exponentials that appear in the expression of $C$, so that its spectral radius can be easily determined.

The general case (system $(*))$ that can be used for any number of patches of hosts and mosquitoes is presented in the Appendix.

The main motivation for the construction of the model here presented as well as the applications made was the recent studies by Forattini et al. ${ }^{4}$ on mosquitoes at an experimental rice irrigation system $^{5,6,7,8,9}$.

The constant and periodic coefficients that appear in the equations of the mathematical model should be determined experimentally. If they are available, reasonable predictions about possible mosquitoborne diseases that can appear at irrigation systems can be made.

\section{ONE PATCH OF HOSTS AND TWO PATCHES OF MOSQUITOES}

A deterministic model is here presented with a view to describing the dynamic of the population of hosts and mosquitoes infected by malaria when there is a homogeneous group of hosts with a constant population $H$ and two groups of mosquitoes of different types $i=1,2$, with population $V_{i}=V_{i}(t)$ periodic in the time $t$ with periodic $T>0$.

Let us considere the following system of ordinary differential equations:

$$
\left\{\begin{array}{l}
\frac{\mathrm{d} S}{\mathrm{~d} t}=-\varepsilon S+\frac{(H-S)}{S}\left(b_{1}^{\prime} f_{1} I_{1}+b_{2}^{\prime} f_{2} I_{2}\right) \\
\frac{\mathrm{d} I_{1}}{\mathrm{~d} t}=-\delta_{1} I_{1}+\left(V_{1}-I_{1}\right) \frac{b_{1} f_{1} S}{V I} \\
\frac{\mathrm{d} I_{2}}{\mathrm{~d} t}=-\delta_{2} I_{2}+\left(V_{2}-I_{2}\right) \frac{b_{2} f_{2} S}{V_{2}}
\end{array}\right.
$$

where:

$H$ : population of hosts;

$V_{i}=V_{i}(t)=V_{i}(t+T)$ : population of mosquitoes at instant $t$, periodic of periodic $T>0$;

$S=S(t)$ : population of infected hosts at instant $t$;

$I_{i}=I_{i}(t)$ : population of infected mosquitoes of type $i$ at instant $t$;

$\delta$ : death rate of mosquitoes of type $i$;

$\xi$ : cure rate of sick hosts;

$b_{i}^{\prime}:$ bites by one mosquito of type $i$ on hosts per unit of time;

$b_{i}=b_{i}(t)$ : bites by mosquitoes of type $i$ taken on one person, per unit of time, which is a periodic function with period $T>0$;

$f_{i}^{\prime} I_{i}$ : population of infected mosquitoes of type $i$ which are infective;

$f_{i} S$ : population of infected hosts which are infective.

Since $b_{i}(t) H=b_{i}^{\prime} V_{i}(t)$, the system above can be written as:

$$
\left\{\begin{array}{l}
\frac{\mathrm{d} S}{\mathrm{~d} t}=-\varepsilon S+\frac{(H-S)}{H}\left(b_{1}^{\prime} f_{1} I_{1}+b_{2}^{\prime} f_{2} I_{2}\right) \\
\frac{\mathrm{d} I_{1}}{\mathrm{~d} t}=-\delta_{1} I_{1}+\left(V_{1}-I_{1}\right) \frac{b_{1}^{\prime} f_{1} S}{H} \\
\frac{\mathrm{d} I_{2}}{\mathrm{~d} t}=-\delta_{2} I_{2}+\left(V_{2}-I_{2}\right) \frac{b_{2}^{\prime} f_{2} S}{H}
\end{array}\right.
$$

where $H, \xi, b_{i}, \delta_{i}, f_{i}$ are positive constants and $V_{i}=V_{i}(t)$ are continuous periodic functions of period $T>0$.

That system (1) corresponds to a generalization of the Ross model (Lotka ${ }^{12}$ ) and of that of DyeHasibeder ${ }^{2,3}$ for malaria. Moreover it is also a variation of the Aronson-Mellander ${ }^{1}$ model that describes the dynamics of gonorrhea.

The main results $A$ and $B$, stated below, for system (1), are special cases of general results proved in the Appendix. 


\section{Theorem A}

Let $\left(S(t), I_{1}(t), I_{2}(t)\right)$ be a non zero solution of $(1)$ such that $0 \leq S\left(t_{0}\right) \leq H, 0 \leq I_{i}\left(t_{0}\right) \leq V_{i}\left(t_{0}\right), i=1,2$, and some $t_{0} \geq 0$. Then $0<S(t)<H, 0<I_{i}(t)<V_{i}(t), i=1,2$, for all $t>t_{0}$.

We may write (1) in a matricial form

$$
\begin{aligned}
& \dot{y}=A(t) y+N(t, y), \quad y=\left(S, I_{1}, I_{2}\right), \text { where } \\
& A(t)=\left(\begin{array}{ccc}
-\xi & b_{1}^{\prime} f_{1} & b_{2}^{\prime} f_{2} \\
\frac{b_{1}^{\prime} f_{1} V_{1}}{H}-\delta_{1} & 0 \\
\frac{b_{2}^{\prime} f_{2} V_{2}}{H} & 0 & -\delta_{2}
\end{array}\right) .
\end{aligned}
$$

Let $\phi(t)$ be the matricial solution of $\dot{y}=A(t) \mathrm{y}$ such that $\phi(0)=I_{d}$. The monodromy matrix $C=\phi(T)$ is positive (Aronson, Mellander ${ }^{1}$, Lemma 2) and, by Perron's theorem (Gantmacher ${ }^{10}$ ), it has a simple positive eigenvalue $\lambda$ such that

$$
\lambda=\max \left\{\operatorname{Re} \lambda_{i}: \operatorname{det}\left(C-\lambda_{i} I_{d}\right)=0\right\}
$$

\section{Theorem B}

There are two possibilities for the non zero solutions $(S(t), I(t))=\left(S(t), I_{1}(t), I_{2}(t)\right)$ of (1) such that for some $t_{0} \geq 0,0 \leq S\left(t_{0}\right) \leq H$ and $0 \leq I_{i}\left(t_{0}\right) \leq V_{i}\left(t_{0}\right), i=1,2$ :

a) If $\lambda \leq 1$ then $(S(t), I(t))$ tends to the zero solution as $t \rightarrow \infty$;

b) If $\lambda>1$, then there exists a unique $T$-periodic solution $\left(S^{*}, I^{*}\right)$ such that for any $t>t_{0}$ we have $0<S^{*}(t)<H$ and $0<I_{i}^{*}(t)<V_{i}(t), i=1,2$. In this case $(S(t)-$ $\left.S^{*}(t), I(t)-I^{*}(t)\right)$ tends to zero as $t \rightarrow \infty$.

In other words, Theorem B says that the infection peters out naturally when $\lambda \leq 1$; or, if $\lambda>1$, the infection becomes endemic if the initial number of infected cases in at least one group is positive.

As usual, it is a non trivial matter to obtain the matrix $C=\phi(T)$ and an expression for $\lambda$.

\section{THE PERIODIC POPULATIONS OF MOSQUITOES AS STEP FUNCTIONS}

\section{One patch of hosts and one patch of mosquitoes}

Let us consider the following system:

$$
\left\{\begin{array}{l}
\frac{\mathrm{d} S}{\mathrm{~d} t}=-\xi S+(H-S) \frac{b^{\prime} f I}{H} \\
\frac{\mathrm{d} I}{\mathrm{~d} t}=-\delta I+(V(t)-I) \frac{b^{\prime} f S}{H}
\end{array}\right.
$$

This describes the dynamic of malaria when we have one group of mosquitoes with a periodic population $V=V(t)$ interacting with a homogeneous group of individuals of a fixed population $H$. It will be shown that for $V=V(t)$ periodic with period $T=$ 12 (months) with $V(t)=V_{i}$ positive and constant, $i<$ $t<(i+1), i=1, \ldots, 12$, the eigenvalues of the fundamental solution of the associated linear system can be computed directly showing their dependence on the data of system (2) that is, on the parameters $\xi$, $\delta, H, b^{\prime}, f$ and on $V=V(t)$.

Let us write (2) in the following form

$$
\left(\begin{array}{l}
\dot{\boldsymbol{S}} \\
\dot{\boldsymbol{I}}
\end{array}\right)=A(t)\left(\begin{array}{l}
S \\
I
\end{array}\right)+N(S, I) \text { where } A(t)=\left(\begin{array}{cc}
-\xi & b^{\prime} f \\
\frac{b^{\prime} f \dot{V}(t)}{H} & -\delta
\end{array}\right)
$$

For each $i=1, \ldots, 12$ one considers $\phi_{i}(t)=\mathrm{e}^{A_{i} t}$, the fundamental solution of

$$
\left(\begin{array}{c}
\dot{S} \\
\dot{I}
\end{array}\right)=A_{i}\left(\begin{array}{l}
S \\
I
\end{array}\right) \text { where } A_{i}=A(i)
$$

If we make $U=-\delta S-b^{\prime} f I$, (2)' becomes

$$
\left(\begin{array}{c}
\dot{S} \\
\dot{U}
\end{array}\right)=\bar{A}_{i}\left(\begin{array}{l}
S \\
U
\end{array}\right), \quad \bar{A}_{i}=\left(\begin{array}{cc}
\operatorname{trace} A_{i} & -1 \\
\operatorname{det} A_{i} & 0
\end{array}\right) .
$$

Since $A_{i}=M \bar{A} M_{i}^{-1}$, with $M=\left(\begin{array}{cc}1 & 0 \\ -\frac{\delta}{b^{\prime} f} & -\frac{1}{b^{\prime} f}\end{array}\right)$ one obtains

$$
\phi_{i}(1)=\mathrm{e}^{A_{i}}=M e^{\bar{A}_{i}} M^{-1} .
$$

Let $\phi(t)$ be the matricial solution of $\left(\begin{array}{l}\dot{S} \\ \dot{I}\end{array}\right)=A(t)\left(\begin{array}{l}S \\ I\end{array}\right)$ such that $\phi(0)=I_{d}$. Then one can write

$$
\begin{aligned}
\phi(T) & =\phi_{12}(1) \phi_{11}(1) \phi_{10}(1) \ldots \phi_{2}(1) \phi_{1}(1) \\
\text { (5) } \quad & =M e^{\overline{A_{12}}} e^{\overline{A_{11}}} e^{\overline{A_{10}}} \ldots e^{\overline{A_{2}}} e^{\overline{A_{1}}} M^{-1} .
\end{aligned}
$$

So from (2)' we have

$$
\ddot{S}-\left(\operatorname{trace} A_{i}\right) \dot{S}+\left(\operatorname{det} A_{i}\right) S=0
$$

and so, from (6)

$$
S(t)=\alpha e^{\lambda_{1}^{i} t}+\beta e^{\lambda_{2}^{i} t}
$$

where the $\lambda_{j}^{i}$ are the real eigenvalues of $A(i)=A_{i}$, $j=1,2, i=1, \ldots, 12$. From (3),

$$
\dot{S}=\left(\operatorname{trace} A_{i}\right) S-U
$$


so we have $U=\alpha \lambda_{2}^{i} e^{\lambda_{1}^{i} t}+\beta \lambda_{1}^{i} e^{\lambda_{2}^{i} t}$ and then

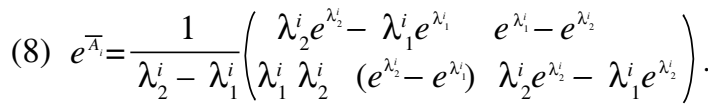

Finally we are able to obtain the eigenvalues of $\phi(T)$ that are those of the following matrix:

$\prod_{i=0}^{11} \frac{1}{\lambda_{2}^{12-i} \lambda_{1}^{12-i}}\left(\begin{array}{cc}\lambda_{2}^{12-i} e^{\lambda_{2}^{12-i}}-\lambda_{1}^{12-i} e^{\lambda_{1}^{12-i}} & e^{\lambda_{1}^{12-i}}-e^{\lambda_{2}^{12-i}} \\ \lambda_{1}^{12-i} \lambda_{2}^{12-i}\left(e^{\lambda_{2}^{12-i}-e^{\lambda_{1}^{12-i}}}\right) & \lambda_{2}^{12-i} e^{\lambda_{1}^{12-1}}-\lambda_{1}^{12-i} e^{\lambda_{2}^{12-i}}\end{array}\right)$

\section{One Patch of Hosts and Two Patches of Mosquitoes}

In this section we consider system (1) with $\delta_{1}=$ $\delta_{2}=\delta$ and $V_{i}=V_{i}(t)$ as a periodic step function, which is positive and constant during the month $i, i=1,2$, $\ldots, 12$, and periodic with period equal to $T=12$ (months). We write

$$
A(t)=\left(\begin{array}{ccc}
-\xi & b_{1}^{\prime} f_{1} & b_{2}^{\prime} f_{2} \\
\frac{b_{1}^{\prime} f_{1}}{H} V_{1} & -\delta & 0 \\
\frac{b_{2}^{\prime} f_{2}}{H} V_{2} & 0 & -\delta
\end{array}\right) .
$$

The three invariants (coefficients of the characteristic polynomium) of $A(t)$ are:

$$
\begin{aligned}
& \operatorname{det} A(t)=-\xi \delta^{2}+\delta \varphi(t) \\
& \operatorname{Tr} A(t)=-\xi-2 \delta(\operatorname{Tr} A(t) \text { does not depend on } t) \\
& \Delta_{2} A(t)=2 \xi \delta+\delta^{2}-\varphi(t)
\end{aligned}
$$

where

$$
\varphi(t)=\frac{\left(b_{1}^{\prime} f_{1}\right)^{2}}{H} V_{1}+\frac{\left(b_{2}^{\prime} f_{2}\right)^{2}}{H} V_{2}
$$

It is easy to check that $(-\delta)$ is a eigenvalue of $A(t)$, say $\lambda_{3}=-\delta$. From the expressions of the invariants, the other two eigenvalues $\lambda_{1}$ and $\lambda_{2}$ satisfy.

$$
\begin{gathered}
\lambda_{1}+\lambda_{2}=-\delta-\xi \\
\lambda_{1} \lambda_{2}=\delta \xi-\varphi(t) .
\end{gathered}
$$

These two last equations imply that

$\lambda_{j}=-\frac{(\delta+\xi)}{2} \pm \frac{1}{2} \sqrt{(\delta-\xi)^{2}+4 \varphi(t)}, \quad \mathrm{j}=1,2$.

Note that the three eigenvalues of $A(t)$ are real and distinct; moreover, we have $\lambda_{2}<-\delta<\lambda_{1}$.
For each $i=1,2, \ldots, 12$, one considers $\phi_{i}(t)=\mathrm{e}^{t A(i)}$ which is the fundamental solution of

$$
\left(\begin{array}{c}
\dot{S} \\
\dot{I}_{1} \\
\dot{I}_{2}
\end{array}\right)=A(i) \quad\left(\begin{array}{l}
S \\
I_{1} \\
I_{2}
\end{array}\right)
$$

If we make

$$
\text { (10) }\left\{\begin{array}{l}
W=-\delta S-b_{2}^{\prime} f_{2} I_{2} \\
U=-2 \delta S-b_{1}^{\prime} f_{1} I_{1}-b_{2}^{\prime} f_{2} I_{2}
\end{array}\right.
$$

system (9) becomes

$$
\left\{\begin{array}{l}
\dot{S}=\operatorname{Tr} A(i) S-U \\
\dot{U}=\left[\delta^{2}+\Delta_{2} A(i)\right] S+\delta U \\
\dot{W}=\left[\delta \xi+\delta^{2}-\frac{\left(b_{2}^{\prime} f_{2}\right)^{2}}{H} V_{2}\right] S+\delta U-\delta W
\end{array}\right.
$$

or $\quad\left(\begin{array}{c}\dot{S} \\ \dot{U} \\ \dot{W}\end{array}\right)=\bar{A}(i) \quad\left(\begin{array}{c}S \\ U \\ W\end{array}\right)$

where $\bar{A}(i)=\left(\begin{array}{ccc}\operatorname{Tr} A(i) & -1 & 0 \\ \delta^{2}+\Delta_{2} A(i) & \delta & 0 \\ \delta \xi+\delta^{2}-\frac{\left(b_{2}^{\prime} f_{2}\right)^{2}}{H} V_{2} & \delta & -\delta\end{array}\right)$.

From (10) we see that $A(i)=M \bar{A}(i) M^{-1}$ with $M^{-1}=$

$$
\left(\begin{array}{ccc}
1 & 0 & 0 \\
-2 \delta & -b_{1}^{\prime} f_{1} & -b_{2}^{\prime} f_{2} \\
-\delta & 0 & -b_{2}^{\prime} f_{2}
\end{array}\right)
$$

Let $\Phi(t)$ be the matricial solution of

$$
\left(\begin{array}{l}
\dot{S}_{\dot{S}} \\
\dot{I}_{1} \\
\dot{I}_{2}
\end{array}\right)=A(t) \quad\left(\begin{array}{l}
S \\
I_{1} \\
I_{2}
\end{array}\right)
$$

such that $I(0)=I_{d}$. Then, as above, one can write

$$
\begin{aligned}
\Phi(T) & =\Phi_{12}(1) \Phi_{11}(1) \ldots \Phi_{2}(1) \Phi_{1}(1) \\
& =M e^{\bar{A}(12)} e^{\bar{A}(11)} \ldots e^{\bar{A}(2)} e^{\bar{A}(1)} M^{-1}
\end{aligned}
$$

Let us write the matrix $\bar{A}(i)$ of system (11) in the form

$$
\bar{A}(i)=\left(\begin{array}{cc}
B(i) & 0 \\
* & -\delta
\end{array}\right)
$$

where $B(i)=\left(\begin{array}{cc}\operatorname{Tr} A(i) & -1 \\ \delta^{2}+\Delta_{2} A(i) & \delta\end{array}\right)$. 
Then

$$
e^{\bar{A}(i)}=\left(\begin{array}{cc}
e^{B(i)} & 0 \\
* & e^{-\delta}
\end{array}\right),
$$

and also

$$
M^{-1} \Phi(T) M=\left(\begin{array}{ccc}
e^{B(12)} e^{B(11)} & \ldots e^{B(1)} & 0 \\
* & & e^{-12 \delta}
\end{array}\right) .
$$

The eigenvalues of $\Phi(T)$ are $e^{-T \delta}$ together with the eigenvalues of $e^{B(12)} e^{B(11)} \ldots e^{B(1)}$. So we only need to compute $e^{B(i)}$ for $i=1, \ldots, 12$. For that one has to solve the system

$$
\left(\begin{array}{ccc}
\dot{S} & S \\
\dot{U} & =B(i) & \\
U
\end{array}\right)
$$

or, equivalently, to solve the second order equation

$$
\ddot{S}-\operatorname{Tr} B(i) \dot{S}+\operatorname{det} B(i) S=0 .
$$

Since the characteristic roots of (13) are the eigenvalues of $B(i)$, that is,

$$
\lambda_{j}^{i}=\lambda_{j}(i)=-\frac{\delta+\xi}{2} \pm \frac{1}{2} \sqrt{(\delta-\xi)^{2}+4 \varphi(i)}
$$

$j=1,2$, and $i=1, \ldots, 12$, then, as we did in section 3.1, $e^{B(i)}$ is given by the second member of equality (8).

\section{APPENDIX}

Let us consider the following system

(*)

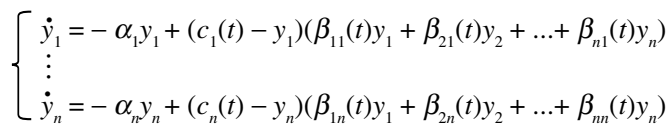

which can be written as

$$
\begin{gathered}
y^{\prime}=A(t) y+N(t, y) \quad \text { where } \\
A(t)=\left(\begin{array}{cccc}
-\alpha_{1}+c_{1} \beta_{11} & c_{1} \beta_{21} & \ldots & c_{1} \beta_{n 1} \\
c_{2} \beta_{12} & -\alpha_{2}+c_{2} \beta_{22} & \ldots & c_{2} \beta_{n 2} \\
\vdots & c_{n} \beta_{2 n} & \ldots & -\alpha_{n}+c_{n} \beta_{n n}
\end{array}\right)
\end{gathered}
$$

Assume $\alpha_{i}$ to be positive constants; $c_{i}=c_{i}(t)$ to be positive continuous periodic functions of period $T>0 ; \beta_{j i}=\beta_{j i}(t)$ to be non negative continuous periodic functions of period $T>0$ such that $A(t)$ is an irreducible matrix (Gantmacher ${ }^{10}$ ), for all $t$. Moreover, assume there exists $\varepsilon>0$ such that $\beta_{j i}(t) \geq$ $\varepsilon$ for all $t$ provided that $\beta_{j i}(t)$ are not identically zero.
For $x=\left(x_{1}, \ldots, x_{n}\right)$ and $y=\left(y_{1}, \ldots, y_{n}\right)$ we denote $x$ $\leq y(x<y)$ if, for all $i, x_{i} \leq y_{i}\left(x_{i}<y_{i}\right)$. With the same arguments used in Aronson, Mellander ${ }^{1}$ one can state:

I) If $y(t)$ and $z(t)$ are non-zero solutions of $(*)$ such that for some $t_{0} \geq 0$ we have $0 \leq y\left(t_{0}\right) \leq z\left(t_{0}\right) \leq c\left(t_{0}\right)$ with $y\left(t_{0}\right) \neq z\left(t_{0}\right)$, then $0<y(t)<z(t)<c(t)$ for all $t>t_{0}$.

For fixed $t_{0} \geq 0$ one considers the map $f_{t_{0}}\left(y_{0}\right)=y\left(t_{0}\right.$ $\left.+T, t_{0}, y_{0}\right)$ for $0 \leq y_{0} \leq c\left(t_{0}\right)$. When $t_{0}=0$ we take the sequence of positive numbers $c(0)=c_{0}>c_{1}>c_{2}>\ldots>$ $c_{n}>\ldots>0$, where $c_{n}=f_{0}\left(\mathrm{c}_{n-1}\right)$ and $\mathrm{Q}=\lim _{n \rightarrow \infty} c_{n} \geq 0$.

When $Q=0$ we have $\lim _{t \rightarrow \infty} y\left(t, t_{0}, y_{0}\right)=0$ for $0 \leq y_{0} \leq c\left(t_{0}\right)$. If $Q \neq 0$ we have $y(t)=y(t, 0, Q)$ positive, periodic with period $T>0$ and, by I), $Q>0$.

Let $\Phi(t)$ the matrix solution of $\dot{y}=A(t) y, \Phi(0)=$ $I_{d}$; since $A(t)=\left(a_{i j}(t)\right)$ is irreducible with $a_{i j}(t) \geq 0$ for $i \neq j$, then the matrix $C=\Phi(T)$ is positive and so, by Perron's theorem, it has a simple positive eigenvalue $\lambda=\max \left\{\operatorname{Re} \lambda_{i} ; \operatorname{det}\left(C-\lambda_{i} I_{d}\right)=0\right\}$.

As in theorem 1 of Aronson, Mellander ${ }^{1}$ one has analogously:

II) If $\lambda_{t-t}<$, there exists $K>0$ such that $|y(t)| \leq$ $K \lambda^{\frac{t-t_{0}}{T}}\left|y\left(t_{0}\right)\right|$ for all $t \geq t_{0} \geq 0$ and any solution $y(t)=y\left(t, t_{0}, y_{0}\right)$ of $\left(^{*}\right)$ such that $0 \leq y_{0} \leq c\left(t_{0}\right)$.

Consider now the case $\lambda>1$. Let $E\left(t_{0}\right)=\left\{y \in \mathbb{R}^{n}\right.$ $\left.: 0 \leq y \leq c\left(t_{0}\right)\right\}, \omega>0$ eigenvector of $C^{t}$ corresponding to $\lambda, v\left(t_{0}\right)^{t}=\omega^{t} \Phi\left(t_{0}\right)^{-1}$ and $E_{\theta}\left(t_{0}\right)=\left\{y \in E\left(t_{0}\right): v\left(t_{0}\right)^{t} y\right.$ $\geq \theta$. We claim that for $\theta>0$ sufficiently small we have $f_{t_{0}}\left(E_{\theta}\left(t_{0}\right)\right) \subset E_{\theta}\left(t_{0}\right)$ where $0 \leq t_{0} \leq T$. In fact

$$
\begin{aligned}
& v\left(t_{0}\right)^{t} f_{t_{0}}(y)=\lambda v\left(t_{0}\right)^{t} y+\varphi\left(t_{0}, y\right) \text { where } \\
& \varphi\left(t_{0}, y\right)=\lambda w^{t} \int_{t_{0}}^{t+T} \Phi^{-1}(s) N\left(s, y\left(s, t_{0}, y\right)\right) \mathrm{d} s ; \text { and } \\
& v\left(t_{0}\right)^{t} f_{t_{0}}(y)-v\left(t_{0}\right)^{t} y=(\lambda-1) v\left(t_{0}\right)^{t} y+\varphi\left(t_{0}, y\right) \geq(\lambda-
\end{aligned}
$$
1) $v^{t} y+\varphi\left(t_{0}, y\right)$, where $0<v \leq v\left(t_{0}\right)$ for all $t_{0} \in[0, T]$. Since

$$
\Phi\left(t_{0}, y\right)=\left\{\begin{array}{cc}
\frac{\varphi\left(t_{0}, y\right)}{|y|} & \text { if } y \neq 0 \\
0 & \text { if } y=0
\end{array}\right.
$$

is continuous on the compact set

$$
\left\{\left(t_{0}, y\right) \mid t_{0} \in[0, T], \mathrm{y} \in E\left(t_{0}\right)\right\},
$$

then there exist $\delta>0$ and $N>0$ such that $v\left(t_{0}\right)^{t} f_{t_{0}}(y)$ $\geq v\left(t_{0}\right)^{t} y+N|y|$ for $t_{0} \in[0, T]$ and $|y| \leq \delta$.

Using the Brower fixed point theorem (Hönig ${ }^{11}$ ) one concludes that $f_{t_{0}}$ has a fixed point in $E_{\theta}\left(t_{0}\right)$. 
For any two solutions $y(t)=y\left(t, t_{0}, y_{0}\right)$ and $z(t)=$ $y\left(t, t_{0}, z_{0}\right)$ of (*) such that $0<y_{0}, z_{0}<c\left(t_{0}\right)$, one has

$$
D^{+} u(\mathrm{t}) \leq-(u-1) \min \left(\psi_{z}, \psi_{y}\right)<0 \text { for } t \geq t_{0} \geq 0
$$

where $u(t)=\max _{k}\left\{\max \left\{\frac{y_{k}}{z_{k}}, \frac{z_{k}}{y_{k}}\right\}\right\}$ and $\psi(y)=$

$$
\min _{1 \leq m \leq n} \sum_{j=1}^{n} \beta_{\mathrm{jm}}(t) y_{j}(t)
$$

(see Aronson and Mellander ${ }^{1}$; Lemma 5, [1]).

So, one can conclude that for $\theta>0$ sufficiently small, $f_{t}: E_{\theta}\left(t_{0}\right) \rightarrow E_{\theta}\left(t_{0}\right)$ has only one fixed point $Q$, and then $f_{t}: E\left(t_{0}\right) \rightarrow E\left(t_{0}\right)$ has only one fixed point $Q$ $>0$, besides the origin, that corresponds to a periodic orbit of period $T>0$ for system $(*)$.

For $y(t)=y\left(t, t_{0}, y_{0}\right)$ with $0 \leq y_{0} \leq c\left(t_{0}\right)$ and $z(t)=$ $y\left(t, t_{0}, Q\right)$ we have

$$
u(t)-1 \leq e^{u\left(t_{o}\right)}\left(u\left(t_{0}\right)-1\right) e^{-p(t)} e^{-\mathrm{a}\left(t-t_{o}\right)}
$$

where $a=\frac{1}{T} \int_{0}^{T} \psi_{z}(t) \mathrm{d} t$ and $p$ is continuous and $T$ periodic, $T>0$. So, for each $x_{0}>0$ sufficiently close to the origin, there exists a constant $M\left(x_{0}\right)>0$ such that

$$
\left|y\left(t, t_{0}, y_{0}\right)-y\left(t, t_{0}, Q\right)\right| \leq M\left(x_{0}\right) e^{-a\left(t-t_{0}\right)}
$$

for all $y_{0}, x_{0} \leq y_{0} \leq c\left(t_{0}\right)$ and all $t \geq t_{0} \geq 0$ because the $c_{k}(t)$

\section{REFERENCES}

1. ARONSON, G. \& MELLANDER, I. A deterministic model in biomathematics. Asymptotic behavior and threshold conditions. Math. Biosci., 49:207-22, 1980.

2. DYE, C \& HASIBEDER, G. Population dynamics of mosquito-borne deseases: effects of flies which bite some peoples more frequently than others. Trans. R. Soc. Trop. Med. Hyg., 80-:69-77, 1986.

3. DYE, C. \& HASIBEDER, G. Population dynamics of mosquitoborne diseases: persistence in a completely heterogeneous environment. Theoret. Pop. Biol., 33:31-53, 1988.

4. FORATTINI, O.P.; KAKITANI, I.; MASSAD, E.; GOMES, A. de C. Studies on mosquitoes (Diptera: Culicidae) and anthropic environment. 1. Parity of blood seeking Anopheles (Kerteszia) in South-Eastern Brazil. Rev. Saúde Pública, 27:1-18, 1993.

5. FORATTINI, O.P.; KAKITANI, I.; MASSAD, E.; MARUCCI, D. Studies on mosquitoes (Diptera: Culicidae) and anthropic environment. 2. Immature stages research at a rice irrigation system location in South-Eastern Brazil. Rev. Saúde Pública, 27:227-36, 1993.

6. FORATTINI, O.P.; KAKITANI, I.; MASSAD, E.; MARUCCI, D. Studies on mosquitoes (Diptera: Culicidae) and anthropic environment. 3. Survey of adult stages at the rice irrigation system and the emergence of Anopheles are bounded functions (see Aronson, Mellander ${ }^{1}$, Th. 2).

Given a compact set $K \subset E\left(t_{0}\right)$, one considers a point $x_{0}, 0<x_{0}<c\left(t_{0}\right)$, such that $x_{0}<y\left(t_{0}+T, t_{0}, y_{0}\right)<$ $c\left(t_{0}\right)$ for all $y_{0} \in K$. Since there are constants $M\left(x_{0}\right)>$ 0 and $N>0$ such that for all $y_{0} \in K$ we have

$$
\left|y\left(t, t_{0}, y_{0}\right)-y\left(t, t_{0}, Q\right)\right| \leq M\left(x_{0}\right) e^{-a\left(t-t_{0}\right)}, t \geq t_{0}+T
$$

and

$$
\left|y\left(t, t_{0}, y_{0}\right)-y\left(t, t_{0}, Q\right)\right| \leq N, t_{0} \leq t \leq t_{0}+T,
$$

then one has the following result.

III) For $\lambda>1$, system $(*)$ admits a unique non zero periodic solution $y\left(t, t_{0}, Q\right)$, which has period $T$, and a constant $a>0$ such that for each compact set $K \subset E\left(t_{0}\right)$ there corresponds a constant $M_{K}>0$ and we have

$$
\left|y\left(t, t_{0}, y_{0}\right)-y\left(t, t_{0}, Q\right)\right| \leq M_{k} e^{-\mathrm{a}\left(t-t_{o}\right)} \text { for all } t \geq t_{0} \geq 0 .
$$

Moreover, as in theorem 3 of Aronson, Mellander ${ }^{1}$ we state:

IV) For $\lambda=1$ there is a constant $L>0$ such that for any solution $y\left(t, t_{0}, y_{0}\right)=y(t)$ of $(*)$ with $0 \leq y_{0} \leq$ $c\left(t_{0}\right), t_{0} \geq 0$, we have

$|y(t)| \leq \frac{K}{1+\left(t-t_{0}\right)}$ provided $t \geq t_{0}$.

albitarsis in South-Eastern Brazil. Rev. Saúde Pública, 27:318-411, 1993.

7. FORATTINI, O.P.; KAKITANI, I.; MASSAD, E.; MARUCCI, D. Studies on mosquitoes (Diptera: Culicidae) and anthropic environment. 4. Survey of resting adults and synanthropic behavior in South-Eastern Brazil. Rev. Saúde Pública, 27:398-411, 1993.

8. FORATTINI, O.P.; KAKITANI, I.; MASSAD, E.; MARUCCI, D. Studies on mosquitoes (Diptera: Culicidae) and anthropic environment. 5. Breeding of Anopheles albitarsis in flooded rice fields in South-Eastern Brazil. Rev. Saúde Pública, 28:329-31, 1994.

9. FORATTINI, O.P.; KAKITANI, I.; MASSAD, E.; MARUCCI, D. Studies on mosquitoes (Diptera: Culicidae) and anthropic environment. 6. Breeding in empty conditions of rice fields in South-Eastern Brazil. Rev. Saúde Pública, 28:395-9, 1994.

10. GANTMACHER, F. R. The theory of matrices. Vol. 2. New York, Chelsea, 1959.

11. HÖNIG, C. S. Aplicações da topologia à análise. IMPA, Rio de Janeiro, 1976.

12. LOTKA, A. J. Contrib. to the analysis of malaria epidemiology. Am. J. Hyg., 3, 1923. 